\title{
An approach to screening for macroprolactinemia in all hyperprolactinemic sera
}

\author{
Nilhan Nurlu Ayan', (ㄷ) Ela Keskin Temeloglu²
}

'Department of Medical Biochemistry, Gaziosmanpasa Taksim Education and Research Hospital, Istanbul, Turkey

${ }^{2}$ Department of Endocrinology and Metabolism Diseases, Gaziosmanpasa Taksim Education and Research Hospital, Istanbul, Turkey

\begin{abstract}
Objectives: Hyperprolactinemia is the most common endocrine disorder of the hypothalamo-pituitary axis and is a result of the over secretion of prolactin (PRL). The presence of the biologically inactive PRL isoform macroprolactin (MPRL) in serum causes interference in the PRL immunoassay, resulting in erroneously high PRL results. The presence of MPRL in serum can be detected using the simple, inexpensive, and routine polyethylene glycol (PEG) precipitation method. This study is an evaluation of the incidence of MPRL in hyperprolactinemic sera and the clinical symptoms and signs of hyper/macroprolactinemic patients.

Methods: The data of 73 hyperprolactinemic patients were retrieved from the laboratory information system and screened for the demographic details, clinical characteristics, and findings of the patients.

Results: Macroprolactinemia was detected in $13.7 \%$ of the patients with hyperprolactinemia. The serum PRL, PRL percentage recovery, and diluted PRL percentage recovery values of macroprolactinemic patients after PEG precipitation were significantly lower than those of hyperprolactinemic patients $(p<0.0001)$. While there was no significant difference in the percentage of infertility or headache cases among the hyperprolactinemic and macroprolactinemic patients, the percentage of oligo/amenorrhea, galactorrhea, and radiological findings (pituitary adenomas) was significantly greater in the hyperprolactinemic patients $(p<0.0001)$.

Conclusion: Measurement of serum MPRL levels using routine PEG precipitation in hyperprolactinemic patients may be very effective in avoiding unnecessary tests and treatments during the diagnosis process and follow-up of patients. Keywords: Hyperprolactinemia, immunoassay, macroprolactin, polyethylene glycol precipitation
\end{abstract}

$\mathrm{H}$ yperprolactinemia is the most common endocrine disorder of the hypothalamus-pituitary axis. It occurs due to extreme prolactin (PRL) secretion. Hyperprolactinemia can be seen in a variety of physiological cases, such as pregnancy, excessive physical activity, stress, and nipple stimulation. The use of dopamine receptor antagonists, antidepressants, antiemetics and opiates can also be pharmacological causes of hyperprolactinemia. However, the most common pathological cause is a PRL-secreting pituitary adenoma (prolactinoma) [1].

Infertility, disorder in the menstrual cycle, oligomenorrhea, amenorrhea, and galactorrhea in women, and oligospermia and impotence in men are among the clinical symptoms of hyperprolactinemia. In some cases, high levels of PRL can be detected in laboratory tests, but the symptoms of hyperprolactinemia are often not observed or are only tenuously observed. Macroprolactin (MPRL), a different form of PRL, can be a cause of hyperprolactinemia [2-5]. The covalently bound dimer "big prolactin" and the much bigger polymeric "big big prolactin" exist in the circulatory system. The monomeric form, MPRL, with a molecular weight of $23.5 \mathrm{kDa}$, makes up $85 \%$ of PRL. MPRL, which is less active biologically, is comprised of "big big" PRL forms [6]. Retrospective analyses have demon-

Address for correspondence: Nilhan Nurlu Ayan, MD. Department of Medical Biochemistry, Gaziosmanpasa Taksim Education and Research Hospital, Istanbul, Turkey

Phone: +90 2122524300 E-mail: nilhannurlu@yahoo.com ORCID: 0000-0002-0844-5050

Submitted Date: August 15, 2018 Accepted Date: October 08, 2018 Available Online Date: January 15, 2019

${ }^{\circ}$ Copyright 2018 by International Journal of Medical Biochemistry - Available online at www.internationalbiochemistry.com

OPEN ACCESS This work is licensed under a Creative Commons Attribution-NonCommercial 4.0 International License. 
strated that approximately $40 \%$ of hyperprolactinemic patients have macroprolactinemia, with a small group of these macroprolactinemic patients displaying hyperprolactinemia symptoms, while $20 \%$ have galactorrhea, $45 \%$ have oligo/ amenorrhea, and $20 \%$ have traces of pituitary adenoma. It has been pointed out that routine MPRL screenings of hyperprolactinemic patients would prevent redundant examinations and treatments, since clinical findings are insufficient to effectively discriminate between hyperprolactinemia and macroprolactinemia, and because macroprolactinemia is the most common reason for hyperprolactinemia [7-9].

Most commercial measurement methods cannot measure MPRL. However, the settling method, which uses polyethylene glycol (PEG), is an inexpensive, easy, and applicable method that is routinely used to test for MPRL in serum [6]. Some studies of immune measurement methods have noted that PEG can cause interference. In order to prevent such interference, it is recommended that laboratory-specific reference intervals be created, with PRL concentrations measured after obtaining settling serums from healthy individuals via PEG, and the dilution of supernatant with a trisaminomethane (TRIS) buffer in a 1 to 5 ratio before analysis. This is due to the fact that some studies have indicated that the measured recovery may exceed $100 \%$ after precipitation with PEG [10-13].

This study examined the frequency of macroprolactinemia symptoms and the consistency of the clinical characteristics of macroprolactinemic patients using laboratory findings. The participants were patients who consulted the Endocrinology and Metabolism Diseases Polyclinic of our hospital and were subsequently diagnosed as hyperprolactinemic.

\section{Materials and Methods}

This was a retrospective, cross-sectional study, and was approved by the Ethics Committee of of the Gaziosmanpasa Taksim Education and Research Hospital (July 08, 2018, protocol:74).

The patients $(n=73)$ were diagnosed with hyperprolactinemia after anamnesis and a physical examination. PRL analysis of serum samples was subsequently performed using a DXI analyzer (Beckman Coulter, Inc., Brea, CA, USA) using the electrochemiluminescence method. Serum MPRL levels of the patients with high levels of PRL (PRL reference interval: 3.34-26.72 $\mathrm{ng} / \mathrm{mL}$ ) were evaluated using the PEG precipitation method, and the results were evaluated in terms of recovery percentage. MPRL measurement in the sera with hyperprolactinemia was performed to diagnose true hyperprolectinemia.

The records of all of the patients who had high serum PRL values and who had MPRL testing performed at our institution between September 2016 and December 2017 were reviewed using the laboratory information system. The demographic details, clinical characteristics, and symptoms of the patients were obtained through the hospital information management system.

\section{Precipitation with polyethylene glycol}

A 25\% PEG solution was prepared by dissolving $25 \mathrm{~g}$ of PEG (polyethylene glycol 6000; Merck Millipore, Burlington, MA, USA) in $100 \mathrm{~mL}$ of physiologic serum.

\section{Macroprolactin study}

A total of $200 \mu \mathrm{l}$ of the patient's serum was added to a $200 \mu \mathrm{l}$ PEG solution, the tube was closed with parafilm, thoroughly mixed in a centrifuge and then left for 15 minutes at ambient temperature. The PRL was subsequently analyzed from the supernatant of the tube, which had been centrifuged at $1500 \mathrm{~g}$ for 30 minutes, and the recovery percentage was counted using the following formula: $M P R L=(P E G$-treated $P L R$ result $\times 2$ / Non-PEG-treated PRL result)x100.

\section{Interpreting the macroprolactin result Recovery percentage}

If the recovery was $>60 \%$, the serum was not considered to include significant MPRL. A result between $40 \%$ and $60 \%$ was accepted as a gray zone, and monitoring was recommended for these patients. A recovery result of $<40 \%$ indicated that the serum included MPRL.

Before the analysis is conducted, dilution of the supernatant using a TRIS buffer at a ratio of 1 to 5 is suggested. This is due to the finding that with certain commercial immune measurement methods used for PRL in laboratories there was an incidence of a recovery of $>100 \%$ after treatment with PEG in analyzers [11]. In this study, the PRL level of 73 hyperprolactinemic case serum samples were analyzed using direct measurements, as well as those of dilution and non-dilution treatment with PEG.

\section{Statistical analysis}

SPSS for Windows, Version 13.0 (SPSS Inc., Chicago, IL, USA) was used to evaluate the data. According to the distributional characteristics of the data, the mean $\pm S D$ (minimum-maximum), or the median and 95\% confidence interval were obtained. In order to compare the parametric data, Student's t-test was used. To compare non-parametric data, the MannWhitney $U$ test was applied, and to evaluate the categorical data, a chi-square test was used. $P<0.05$ was accepted as statistically significant.

\section{Results}

Macroprolactinemia was determined in $10(13.7 \%)$ of 73 hyperprolactinemic patients. As seen in Table 1, the age, body mass index, and gender characteristics of the hyperprolactinemic patients were not significantly different from those of the macroprolactinemic patients. However, the serum PRL, dilution, and non-dilution PRL recovery percentage values of the macroprolactinemic patients after treatment with PEG were significantly lower than those of the hyperprolactinemic patients $(p<0.0001)$. 
Table 2 illustrates the age and gender characteristics of macroprolactinemic patients, the serum direct PRL, PRL after treatment with PEG, and recovery percentage values, as well as related symptoms and findings.
In Table 3, the percentage comparisons of hyperprolactinemic and macroprolactinemic patients in terms of hyperprolactinemia symptoms and findings are provided. While no significant differences were found between the hyperprolactinemic and

Table 1. Clinical characteristics and prolactin measurement results of patients

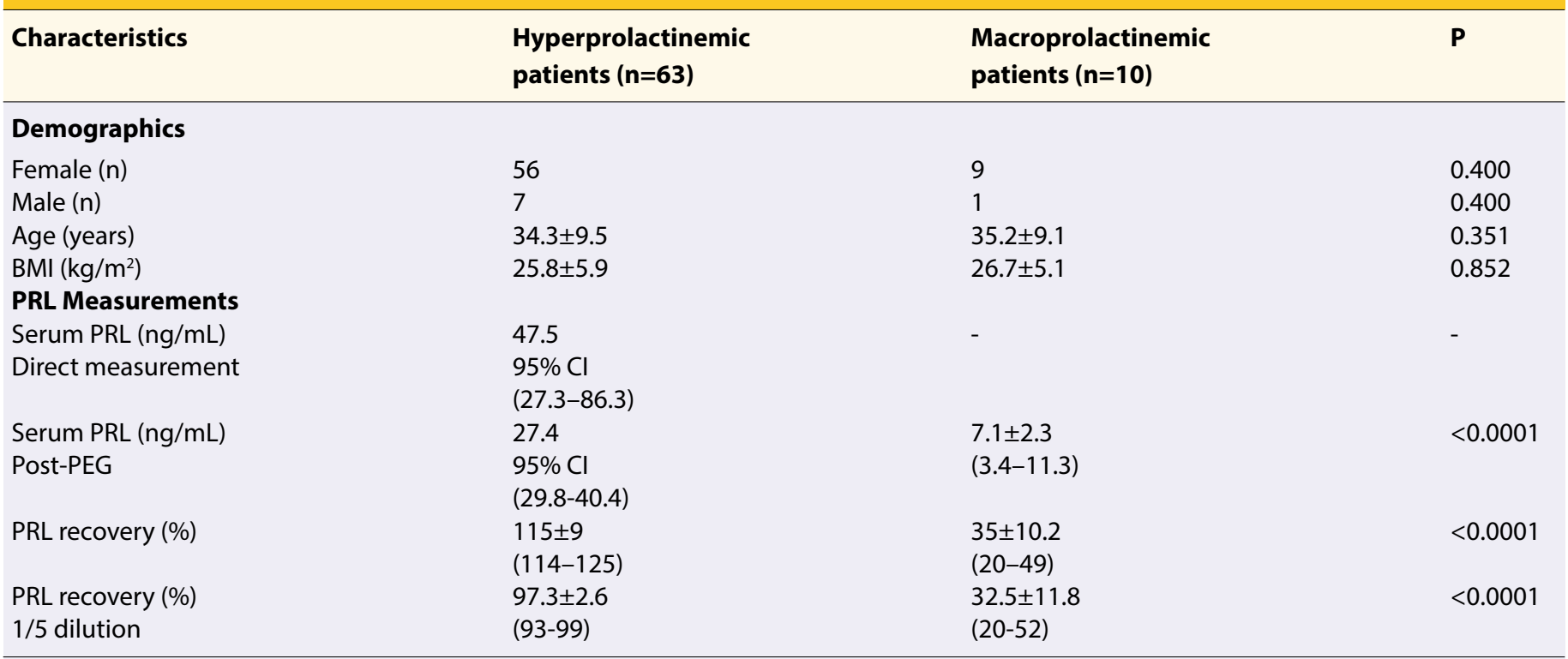

BMI:Body mass Index; PEG: Polyethylene glycol; PRL: Prolactin.

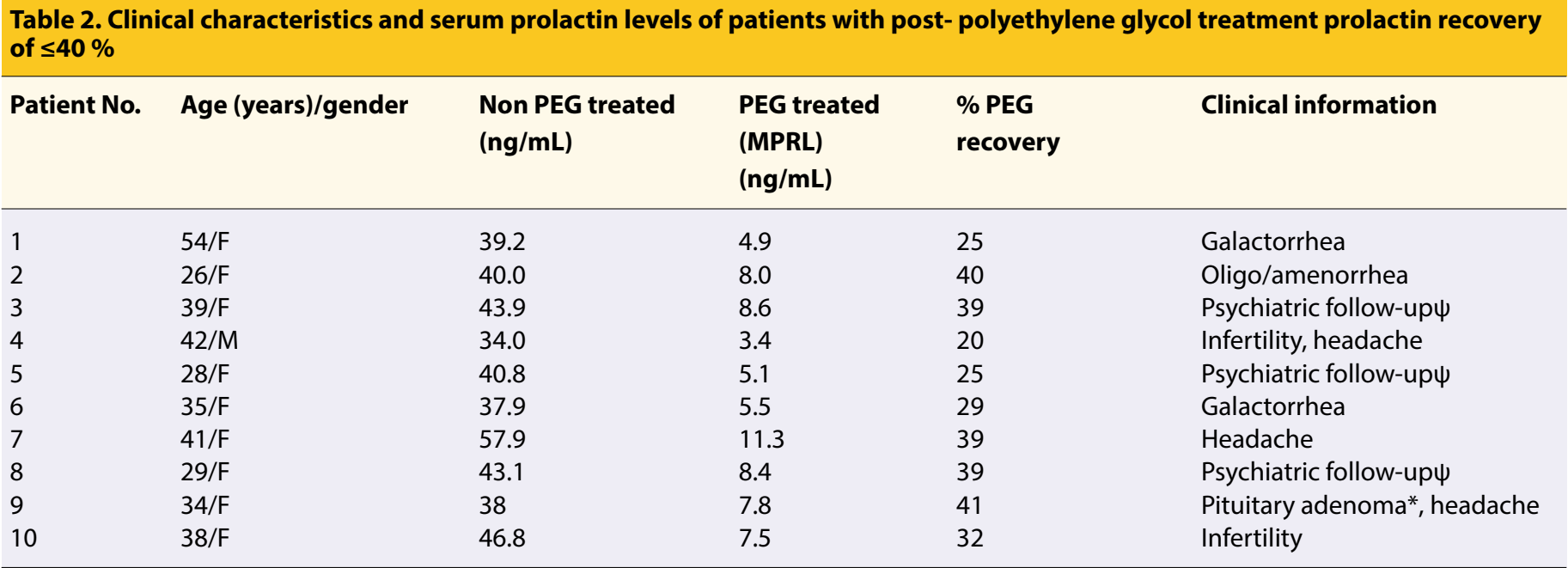

*MRI scan; $\psi$ patients using antidepressant.

F: Female; M: Male; MPRL: Macroprolactin; PEG: Polyethylene glycol; PRL: Prolactin.

Table 3. Comparison of symptoms and findings of hyperprolactinemic and macroprolactinemic patients

\begin{tabular}{lllc}
\hline Symptom and findings & $\begin{array}{l}\text { Hyperprolactinemic } \\
\text { patients } \mathbf{n = 6 3}\end{array}$ & $\begin{array}{l}\text { Macroprolactinemic } \\
\text { patients } \mathbf{n = 1 0}\end{array}$ & $\begin{array}{l}\mathbf{P} \\
\text { Oligo/amenorrhea (\%) }\end{array}$ \\
Galactorrhea (\%) & 46 & 10 & $<0.0001$ \\
Infertility (\%) & 38 & 20 & $<0.0001$ \\
Headache (\%) & 14 & 20 & 0.259 \\
Radiological finding (\%) & 38 & 30 & 0.232 \\
& 48 & 10 & $<0.0001$
\end{tabular}


macroprolactinemic patients in terms of the incidence of infertility and headache, the percentage of oligo/amenorrhea, galactorrhea, and radiological findings (pituitary adenoma) was significantly greater in the hyperprolactinemic patients $(p<0.0001)$.

\section{Discussion}

Pituitary adenoma is the most commonly encountered cause of excess PRL release, and prolactinoma is the most often seen endocrine disorder of the hypothalamo-pituitary axis. The direct clinical results of an extreme release of PRL may include hypogonadism and galactorrhea. The first thing to be done when dealing with suspected hyperprolactinemia after a thorough anamnesis and physical examination is to evaluate serum PRL levels. However, laboratories face difficulties when taking PRL measurements based on the existing methods of distinguishing macroprolactinemia from true hyperprolactinemia $[6,11]$. In spite of efforts made to improve test specificity, all PRL immune measurement methods in routine use measure different levels of MPRL. This can lead to an incorrect hyperprolactinemia diagnosis and further radiological imaging and/or unnecessary treatments $[10,12,13]$.

Although gel filtration chromography (GFC) is a reference method for monomeric PRL measurement in serum, the method is not used routinely in laboratories, as it is slow, requires intensive labor, and is expensive $[9,14-16]$. Numerous studies have compared the PEG precipitation method with GFC in macro/ hyperprolactinemia analysis [10-15]. Since GFC is not available for the measurement of PRL in our country, confirmation of hyperprolactinemic sera with GFC could not be performed for the present study.

MPRL determination with the PEG precipitation method is widely used in laboratories as an easily applicable and inexpensive method $[17,18]$. However, there are some disadvantages to the PEG precipitation method. Some studies have indicated that there was PEG interference in routine immunoassays using analyzers such as the AxSym (Abbott Laboratories, Inc., Lake Bluff, IL, USA), the Access 2 (Beckman Coulter, Inc., Brea, CA, USA), and the Immulite 2000 (Siemens Healthineers AG, Erlangen, Germany) $[11,13,19,20]$. It is recommended that such potential interference be anticipated and interpreted and that the sera of blood of healthy volunteers also be treated with PEG to form individual reference intervals for each laboratory $[10-12,15]$. The total PRL concentration of a serum, including MPRL, are to be placed into a PRL reference interval and identified after treatment with PEG. This means that the laboratory will easily be able to confirm a macroprolactinemia diagnosis. There are plans to use healthy volunteer serum with PEG and to thereby determine PRL reference intervals after treatment with PEG for our laboratory. We also believe that, as a result of this study, contributions can be made to clinician interpretation by reporting PRL values $(\mathrm{ng} / \mathrm{mL})$, in addition to recovery percentage, after treatment with PEG.

When screening was performed using the MPRL using the PEG precipitation method under laboratory conditions, it was observed that recovery was always $>100 \%$ in sera that did not include MPRL. Our research confirmed that the recovery measured after treatment with PEG can be over $100 \%$ in some commercial immune measurement analyzers. It was also confirmed that this interference can be eliminated by dilution of the supernatant with a TRIS buffer in a 1 to 5 ratio before the analysis [11]. As indicated in Table 1, the PEG interference was eliminated by $P R L$ recovery after dilution of the supernatant of PEG-treated serum with a $1 / 5$ TRIS buffer. A statistically significant difference was found between the diluted and non-diluted results of PEGtreated serum with a TRIS buffer when examining for hyperprolactinemia and macroprolactinemia.

McCudden et al.[9] reported in their study that MPRL is a biologically inactive form that commonly does not cause hyperprolactinemia symptoms and findings. The authors also noted that no difference was found between hyperprolactinemic patients with and without MPRL in terms of oligo/amenorrhea, galactorrhea, and abnormal magnetic resonance imaging (MRI) results [21]. However, a significant difference was observed in this study between macroprolactinemic and hyperprolactinemic patients in terms of the incidence of oligo/amenorrhea, galactorrhea symptoms, and MRI findings $(p<0.0001)$. The infertility and headache incidence percentages were similar in both the macroprolactinemic and the hyperprolactinemic patients. This result may be explained by macroprolactinemia often occurring in patients with a high monomeric PRL value.

MPRL evaluation is recommended in patients with asymptomatic hyperprolactinemia. MPRL is usually bioinactive and has a low capacity to bind to the receptor in target organs. Therefore, unlike true hyperprolactinemia, patients with macroprolactinemia are usually resistant to the treatment with a dopamine agonist (cabergoline) $[6,22,23]$. One study has shown that there was no significant difference between the MPRL values measured using the PEG precipitation method before and after dopamine agonist treatment [24]. Therefore, if the cause of hyperprolactinemia is macroprolactinemia, there may be the question of the application of unnecessary treatment and related costs.

Smith et al.[13] stated that among the analysis systems they examined, some immunoassay systems were superior in providing normoprolactinemic values in all sera that were known to include MPRL. However, as a result of an inability to determine the level of success, secondary screening was recommended. Creating convenient criteria for MPRL screening using a PEG precipitation method depends on the analytic system used [25].

\section{Conclusion}

The appropriate criteria to obtain accurate and comparable PRL test results and to create a basis for mutual recognition occurring between different laboratories should be developed by clinicians and laboratory physicians.

Conflict of interest: There is no conflict of interest between the authors. 
Ethics Committee Approval: The ethics committee approval of this study was approved by the Ethics Committee of the GOP Taksim Training and Research Hospital with decision no. 74 of 08/08/2018.

Financial Disclosure: Macroprolactin screening was deemed appropriate for every serum determined in our laboratory by the decision of the GOP Taksim Training and Research Hospital Laboratory Services and Analysis Commission No. 3, which was taken on 23.05.2016 by the need of macroprolactin treatment for the patients whose endocrinology and metabolic disease clinics doctors were determined to have elevated PRL. and HBYS has been opened on the request page at the request of the relevant physicians.

Peer-review: Externally peer-reviewed.

Authorship contributions: Concept - N.N.A., E.K.T.; Design N.N.A., E.K.T.; Supervision - E.K.T.; Materials - N.N.A., E.K.T.; Data collection \&/or processing - N.N.A.; Analysis and/or interpretation - N.N.A., E.K.T.; Literature search - N.N.A.; Writing - N.N.A.; Critical review - E.K.T.

\section{References}

1. Melmed S, Kleinberg D. Anterior pituitary. In: Kronenberg HM, Melmed S, Polonsky KS, Larsen PR, editors. Williams textbook of endocrinology. 11th ed. Philadelphia: Saunders Elsevier; 2008. p. 185-261.

2. Klibanski A. Clinical practice. Prolactinomas. N Engl J Med 2010;362:1219-26. [CrossRef]

3. Elenkova A, Genov N, Abadzhieva Z, Kirilov G, Vasilev V, Kalinov $\mathrm{K}$, et al. Macroprolactinemia in patients with prolactinomas: prevalence and clinical significance. Exp Clin Endocrinol Diabetes 2013;121:201-5. [CrossRef]

4. Leite V, Cosby H, Sobrinho LG, Fresnoza MA, Santos MA, Friesen HG. Characterization of big, big prolactin in patients with hyperprolactinaemia. Clin Endocrinol (Oxf) 1992;37:365-72. [CrossRef]

5. Jackson RD, Wortsman J, Malarkey WB. Characterization of a large molecular weight prolactin in women with idiopathic hyperprolactinemia and normal menses. J Clin Endocrinol Metab 1985;61:258-64. [CrossRef]

6. Melmed S, Casanueva FF, Hoffman AR, Kleinberg DL, Montori VM, Schlechte JA, et al; Endocrine Society. Diagnosis and treatment of hyperprolactinemia: an Endocrine Society clinical practice guideline. J Clin Endocrinol Metab 2011;96:273-88.

7. Donadio F, Barbieri A, Angioni R, Mantovani G, Beck-Peccoz P, Spada A, et al. Patients with macroprolactinaemia: clinical and radiological features. Eur J Clin Invest 2007;37:552-7. [CrossRef]

8. McKenna TJ. Should macroprolactin be measured in all hyperprolactinaemic sera? Clin Endocrinol (Oxf) 2009;71:466-9.

9. Gibney J, Smith TP, McKenna TJ. Clinical relevance of macroprolactin. Clin Endocrinol (Oxf) 2005;62:633-43. [CrossRef]

10. Ellis MJ, Livesey JH, Soule SG. Macroprolactin, big-prolactin and potential effects on the misdiagnosis of hyperprolactinemia using the Beckman Coulter Access Prolactin assay. Clin Biochem 2006;39:1028-34. [CrossRef]
11. Beltran L, Fahie-Wilson MN, McKenna TJ, Kavanagh L, Smith TP. Serum total prolactin and monomeric prolactin reference intervals determined by precipitation with polyethylene glycol: evaluation and validation on common immunoassay platforms. Clin Chem 2008;54:1673-81. [CrossRef]

12. Suliman AM, Smith TP, Gibney J, McKenna TJ. Frequent misdiagnosis and mismanagement of hyperprolactinemic patients before the introduction of macroprolactin screening: application of a new strict laboratory definition of macroprolactinemia. Clin Chem 2003;49:1504-9. [CrossRef]

13. Smith TP, Suliman AM, Fahie-Wilson MN, McKenna TJ. Gross variability in the detection of prolactin in sera containing big big prolactin (macroprolactin) by commercial immunoassays. J Clin Endocrinol Metab 2002;87:5410-5. [CrossRef]

14. Prazeres S, Santos MA, Ferreira HG, Sobrinho LG. A practical method for the detection of macroprolactinaemia using ultrafiltration. Clin Endocrinol (Oxf) 2003;58:686-90. [CrossRef]

15. Kavanagh L, McKenna TJ, Fahie-Wilson MN, Gibney J, Smith TP. Specificity and clinical utility of methods for the detection of macroprolactin. Clin Chem 2006;52:1366-72. [CrossRef]

16. Gibney J, Smith TP, McKenna TJ. The impact on clinical practice of routine screening for macroprolactin. J Clin Endocrinol Metab 2005;90:3927-32. [CrossRef]

17. Vieira JG, Tachibana TT, Obara LH, Maciel RM. Extensive experience and validation of polyethylene glycol precipitation as a screening method for macroprolactinemia. Clin Chem 1998;44:1758-9.

18. Fahie-Wilson MN. Polyethylene glycol precipitation as a screening method for macroprolactinemia. Clin Chem 1999;45:436-7.

19. Schneider W, Marcovitz S, Al-Shammari S, Yago S, Chevalier S. Reactivity of macroprolactin in common automated immunoassays. Clin Biochem 2001;34:469-73. [CrossRef]

20. Gilson G, Schmit P, Thix J, Hoffman JP, Humbel RL. Prolactin results for samples containing macroprolactin are method and sample dependent. Clin Chem 2001;47:331-3.

21. McCudden CR, Sharpless JL, Grenache DG. Comparison of multiple methods for identification of hyperprolactinemia in the presence of macroprolactin. Clin Chim Acta 2010;411:155-60.

22. Cavaco B, Leite V, Santos MA, Arranhado E, Sobrinho LG. Some forms of big big prolactin behave as a complex of monomeric prolactin with an immunoglobulin $\mathrm{G}$ in patients with macroprolactinemia or prolactinoma. J Clin Endocrinol Metab 1995;80:2342-6. [CrossRef]

23. Hattori N, Ikekubo K, Ishihara T, Moridera K, Hino M, Kurahachi $\mathrm{H}$. Correlation of the antibody titers with serum prolactin levels and their clinical course in patients with anti-prolactin autoantibody. Eur J Endocrinol 1994;130:438-45. [CrossRef]

24. Lu CC, Hsieh CJ. The importance of measuring macroprolactin in the differential diagnosis of hyperprolactinemic patients. Kaohsiung J Med Sci 2012;28:94-9. [CrossRef]

25. Chen YJ, Song GZ, Wang ZN. A new criteria for screening macroprolactinemia using polyethylene glycol treatment combined with different assays for prolactin. Eur Rev Med Pharmacol Sci 2016;20:1788-94. 